\title{
ANÁLISE DOS INDICADORES DE PRODUTIVIDADE DOS CENTROS DE ATENÇÃO PSICOSSOCIAL ENTRE AS MACRORREGIÕES DO ESTADO DA PARAÍBA NOS ANOS DE 2013 A 2020
}

\author{
ANALYSIS OF THE PRODUCTIVITY INDICATORS OF \\ PSYCHOSOCIAL ATTENTION CENTERS AMONG THE \\ MACROREGIONS OF THE STATE OF PARAIIBA IN THE YEARS OF \\ 2013 TO 2020
}

Renato Américo Dantas Camilo de Souza1 Jonathan Bento Cavalcanti ${ }^{2}$ Edwirde Luiz Silva Camêlo ${ }^{3}$

\begin{abstract}
RESUMO: OBJETIVO: Este estudo buscou rastrear e discutir as prováveis correlações estatísticas observadas entre alguns dos principais indicadores de produtividade dos serviços de saúde mental oferecidos pelos CAPS das 4 macrorregiões do estado da Paraíba. METODOLOGIA: Trata-se de um estudo descritivo- analítico, baseado em dados secundários registrados entre Janeiro de 2013 e Dezembro de 2020 nas bases de dados governamentais do Sistema de Informações Hospitalares do Departamento de Informática do Sistema Único de Saúde, SIH-DATASUS. RESULTADOS: Com exceçãodos atendimentos em grupo, a quantidade de procedimentos realizados nos CAPS da regiãoda Mata paraibana demonstra pouquíssima dispersão, o que aponta para uma constância no decorrer dos anos. Os atendimentos em grupo nessa região também aparecem de forma um pouco simétrica, o valor máximo foi puxado por um ponto extremo, mostrando que a Mata Paraibana tem pontos elevados por causa das grandes cidades e da densidade populacional. CONCLUSÃO: Reitera-se a necessidade de defesa do SUS enquanto pauta política fundamental à consolidação da garantia de direito ao acesso amplo e democrático da população à serviços desaúde mental humanizados, especializados e de alta qualidade.
\end{abstract}

\footnotetext{
1 Graduando de Psicologia pela Universidade Estadual da Paraíba - UEPB, macrenato2010@gmail.com.

2 Graduando de Psicologia pela Universidade Estadual da Paraíba - UEPB, jonathan.ifpb@gmail.com.

3 Professor de Estatística pela Universidade Estadual da Paraíba - UEPB, edwirde@uepb.edu.br.
} 
Palavras chave: Centro de Atenção Psicossocial. Psicologia da Saúde. Políticas Públicas. Sistema Único de Saúde.

ABSTRACT: OBJECTIVE: This study sought to track and discuss the probable statistical correlations observed among some of the main indicators of productivity of mental health services offered by CAPS in the 4 macro-regions of the state of Paraíba. METHODOLOGY: This is a descriptive-analytical study, based on secondary data recorded between January 2013 and December 2020 in the government databases of the Hospital Information System of the Informatics Department of the Unified Health System, SIH-DATASUS. RESULTS: With the exception of group visits, the number of procedures performed at CAPS in the region of Mata Paraibana shows very little dispersion, which points to a constancy over the years. Group visits in this region also appear somewhat symmetrical, the maximum value was pulled by an extreme point, showing that Mata Paraibana has high points because of large cities and population density. CONCLUSION: The need to defend SUS as a fundamental political agenda for the consolidation of the guarantee of the right to broad and democratic access of the population to humanized, specialized and high quality mental health services is reiterated.

Keywords: Psychosocial Care Center. Health Psychology. Public Policies. Health Unic System. 


\section{INTRODUÇÃO}

O Sistema Único de Saúde-SUS foi regulamentado em 1990 por meio das Leis Orgânicas da Saúde - Lei n. 8.080/1990 e Lei n. 8.142/1990. As pressões de vários setores da sociedade pela aquisição de direitos civis foi justamente o que culminou na promulgação da Constituição Federal de 1988, berço dos princípios doutrinários da universalidade, equidade e integralidade, ambos basilares para 0 SUS (SALES et al, 2019).

Inspirada por um movimento semelhante ocorrido na Itália e encabeçado por Franco Basaglia, a partir da década de 1980 ocorre no Brasil o movimento de Reforma Psiquiátrica em oposição ao modelo asilar até então amplamente empregado e que consistia no isolamento, exílio e extermínio das pessoas em sofrimento mental e daqueles considerados subversivos (GODOI, 2020), sendo assim responsável pela mudança de como a saúde mental vinha sendo tratada no país. Mesmo com algumas poucas experiências com modelos substitutivos ao asilar que precederam a promulgação da Lei Paulo Delgado no final da década de 1980, foi apenas a partir desta que os direitos da pessoa com transtornos mentais e a extinção dos manicômios começou a ser discutida e aprovada no âmbito legislativo (AMARANTE, 1994).

Após a instituição do SUS, criaram-se diversos níveis de atenção social, entre eles a Atenção Primária em Saúde, que serve como principal porta de entrada para o sistema. O nível primário é de vital importância para um cuidado efetivo com a saúde mental, constituindo um lugar estratégico para todos os trabalhos do psicólogo (GODOI, 2020). Exercendo sua função por meio de oficinas, rodas de conversas, ações socioeducativas, atenção às crises, acolhimento e demais estratégias de prevenção e promoção em saúde, o fazer do psicólogo no SUS, além de contribuir com a equipe multiprofissional, compõe-se na prática através de atendimentos individuais e grupais no âmbito comunitário, domiciliar e hospitalar, dentre outros (CAMPOS \& GUARIDO, 2010; RIBEIRO, 2011). 
Atualmente, quase 15 mil psicólogos trabalham no SUS, tendo conquistado umainserção maior à medida que a saúde pública se desenvolve no país (CAMPOS \& GUARIDO, 2010). Isso foi possível, em grande parte, graças ao desenvolvimento da Rede de Atenção Psicossocial-RAPS, que integrou todos os níveis de atenção à saúde, destacando-se no campo da saúde mental o Núcleo de Apoio à Saúde da Família-NASF e os Centros de Atenção Psicossocial-CAPS. Enquanto o NASF é composto por uma equipe multiprofissional de nível superior, compartilhando práticas e saberes em saúde dos territórios sob suas respectivas responsabilidades para poder atuar de forma integrada, os CAPS atuam porterritório diretamente com as comunidades oferecendo uma superação do modelo biomédico e asilar, se subdividindo em diversos tipos de unidades, com focos e estruturas diferentes (GODOI, 2020; GOMES, 2013).

Este estudo buscou rastrear e discutir as prováveis correlações estatísticas observadas entre alguns dos principais indicadores de produtividade dos serviços de saúde mental oferecidos pelos CAPS das 4 macrorregiões do estado da Paraíba, disponibilizadas nas bases de dados governamentais vinculadas ao SUS. A presente investigação se propõe a contribuir para o avanço das discussões acerca dos desdobramentos político-institucionais que pautam a inserção do CAPS enquanto espaço de produção de saúde e qualidade de vida nos múltiplos contextos sociais do estado.

\section{METODOLOGIA}

Conduziu-se uma avaliação de caráter descritivo, explicativo e quantitativo, a partir dos dados secundários registrados entre Janeiro de 2010 e Dezembro de 2020 no Sistema de Informações Ambulatoriais do Departamento de Informações do Sistema Único de Saúde - SIA/DATASUS, e coletados através do aplicativo de tabulação de dados TabNet. Foram incluídas no estudo as quantidades registradas referentes aos seguintes procedimentos: "Acolhimento inicial"; "Atendimento individual"; "Atendimento em grupo"; "Atendimento familiar" e "Atendimento 
domiciliar". Para cada uma das 5 variáveis acima apontadas, consideraram-se os recortes específicos das 4 mesorregiões do estado da Paraíba: "Sertão paraibano"; "Borborema"; "Agreste paraibano" e "Mata paraibana".

Os dados quantitativos coletados no SIH/DATASUS foram tabulados em planilhas por meio do programa Microsoft Excel 2016 no sistema operacional Windows 8. Para fins de processamento e avaliação das informações, lançou-se mão da estatística descritiva, optando pela representação gráfica boxplot para análise de dispersão. O boxplot oferece uma representação gráfica de dados através de seus resumos de cinco números. A linha vertical dentro da caixa é a mediana $\left(Q_{2}\right)$, a linha vertical do lado esquerdo da caixa representa a localização $Q_{1}$ (primeiro quartil) e a linha vertical do lado direito da caixa representa a localização de $Q_{3}$ (terceiro quartil). Portanto a caixa contém as observações entre $25 \%$ e $75 \%$ da distribuição das quantidades, ou seja, os $50 \%$ centrais. Os $25 \%$ inferiores das quantidades estão representados por uma linha vertical que liga o lado esquerdo da caixa ao menor valor. Os pontos isolados muito acima ou muito abaixo da caixa pode ser considerado com outliers.

Essa análise foi realizada através do programa $R$ (2019). Foram utilizados os seguintes pacotes no R: ade4 (Bougeard S, Dray S (2018)), corrplot (Taiyun Wei and Viliam Simko (2017).

Ainda para o fim da descrição e análise dos dados, foram utilizadas tabelas. Por se tratarem de informações governamentais de domínio público sem identificação de indivíduos, o presente estudo exime-se de apreciação por comitê de ética.

\section{RESULTADOS}

Com uma população estimada em 4.039,277 habitantes (IBGE, 2020), o estado da Paraíba é a quinta Unidade Federativa mais populosa do Nordeste brasileiro, sendo formada por 223 municípios distribuídos entre 4 mesorregiões e 23 microrregiões. Em 1976, as mesorregiões foram criadas para fins estatísticos 
enquanto um agrupamento espacial intermediário entre microrregiões relativamente homogêneas, subdividindo estados emunicípios em áreas geográficas que mantém entre si algum grau de similaridade socioeconômica, embora não constituam uma entidade própria de natureza política ou administrativa (QUEIROZ, 2017).

Figura 1: Mapa de mesorregiões do Estado da Paraíba.

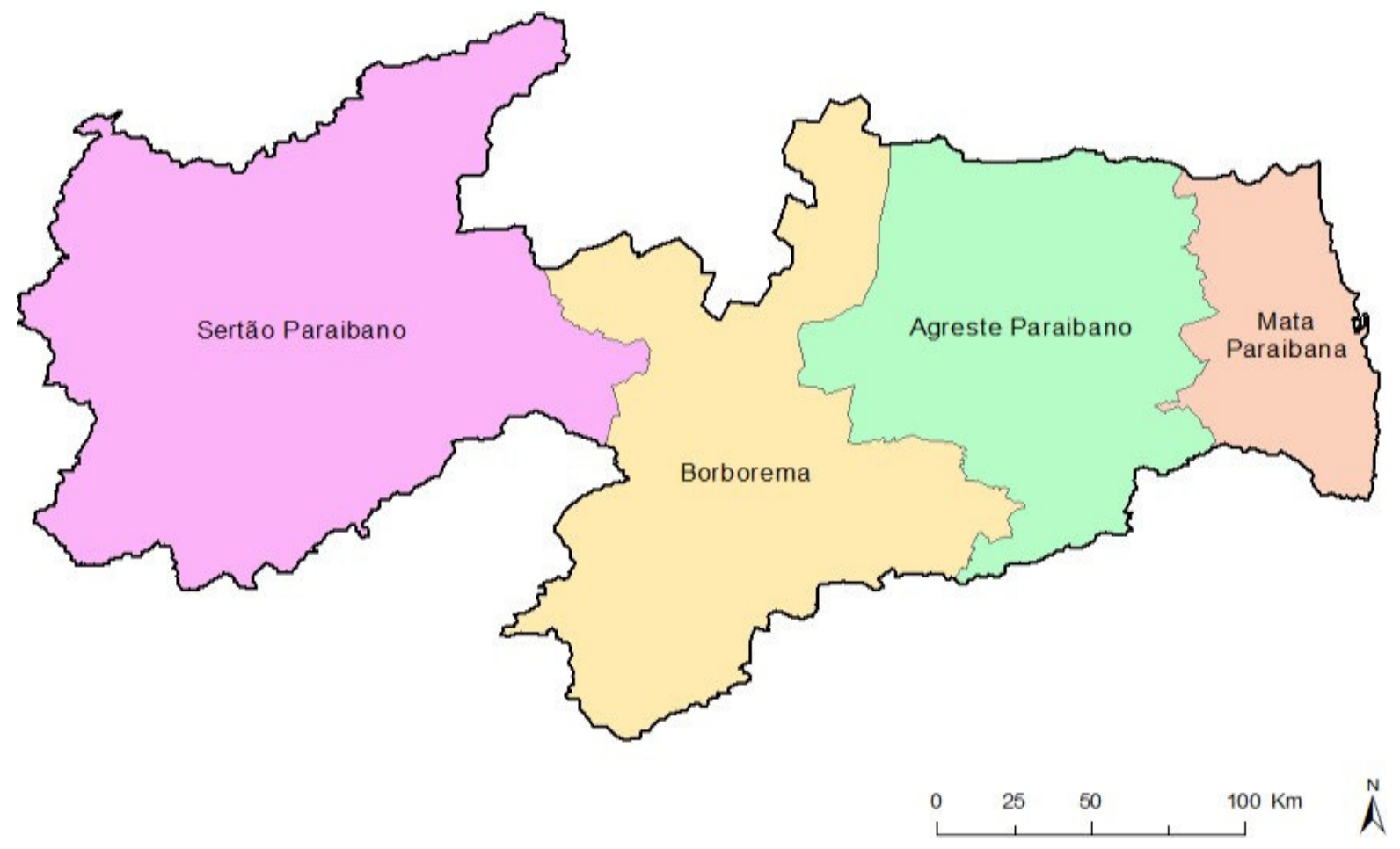

Fonte: IBGE (2017). 
Infográfico populacional entre as mesorregiões e microrregiões do Estado da Paraíba.

\begin{tabular}{|c|c|c|c|}
\hline MESORREGIÃO & MICRORREGIÃO & N - Micro & N - Meso \\
\hline Agreste Paraibano & $\begin{array}{l}\text { Brejo Paraibano } \\
\text { Campina Grande } \\
\text { Curimataú Ocidental } \\
\text { Curimataú Oriental } \\
\text { Esperança } \\
\text { Guarabira } \\
\text { Itabaiana } \\
\text { Umbuzeiro }\end{array}$ & $\begin{array}{c}115.731 \\
536.714 \\
129.109 \\
96.368 \\
57.692 \\
171.646 \\
111.661 \\
54.322\end{array}$ & 1.273 .243 \\
\hline Borborema & $\begin{array}{c}\text { Cariri Ocidental } \\
\text { Cariri Oriental } \\
\text { Seridó Ocidental Paraibano } \\
\text { Seridó Oriental Paraibano }\end{array}$ & $\begin{array}{c}128.799 \\
67.264 \\
41.242 \\
79.590 \\
\end{array}$ & 316.895 \\
\hline Mata Paraibana & $\begin{array}{c}\text { João Pessoa } \\
\text { Litoral Norte } \\
\text { Litoral Sul } \\
\text { Sapé }\end{array}$ & $\begin{array}{c}1.159 .054 \\
153.223 \\
89.490 \\
140.664\end{array}$ & 1.542 .431 \\
\hline Sertão Paraibano & $\begin{array}{c}\text { Cajazeiras } \\
\text { Catolé do Rocha } \\
\text { Itaporanga } \\
\text { Patos } \\
\text { Piancó } \\
\text { Serra do Teixeira } \\
\text { Sousa }\end{array}$ & $\begin{array}{c}178.104 \\
123.679 \\
85.901 \\
135.227 \\
71.129 \\
122.625 \\
190.043\end{array}$ & 906.708 \\
\hline
\end{tabular}

Fonte: IBGE (2020).

A Figura 2 mostra uma maneira conveniente de descrever tanto o centro como a dispersão de um conjunto de dados, consistindo em tomar a mediana para determinar o centroe os quartis para determinar a dispersão entre o menor e o maior valor. O espaçamento dos quartis e dos extremos em relação à mediana dá uma indicação da simetria ou da assimetriada distribuição. 
Figura 2: Dispersão gráfica das quantidades totais para as cinco variáveis, distribuídas entreas quatro mesorregiões do estado da Paraíba.
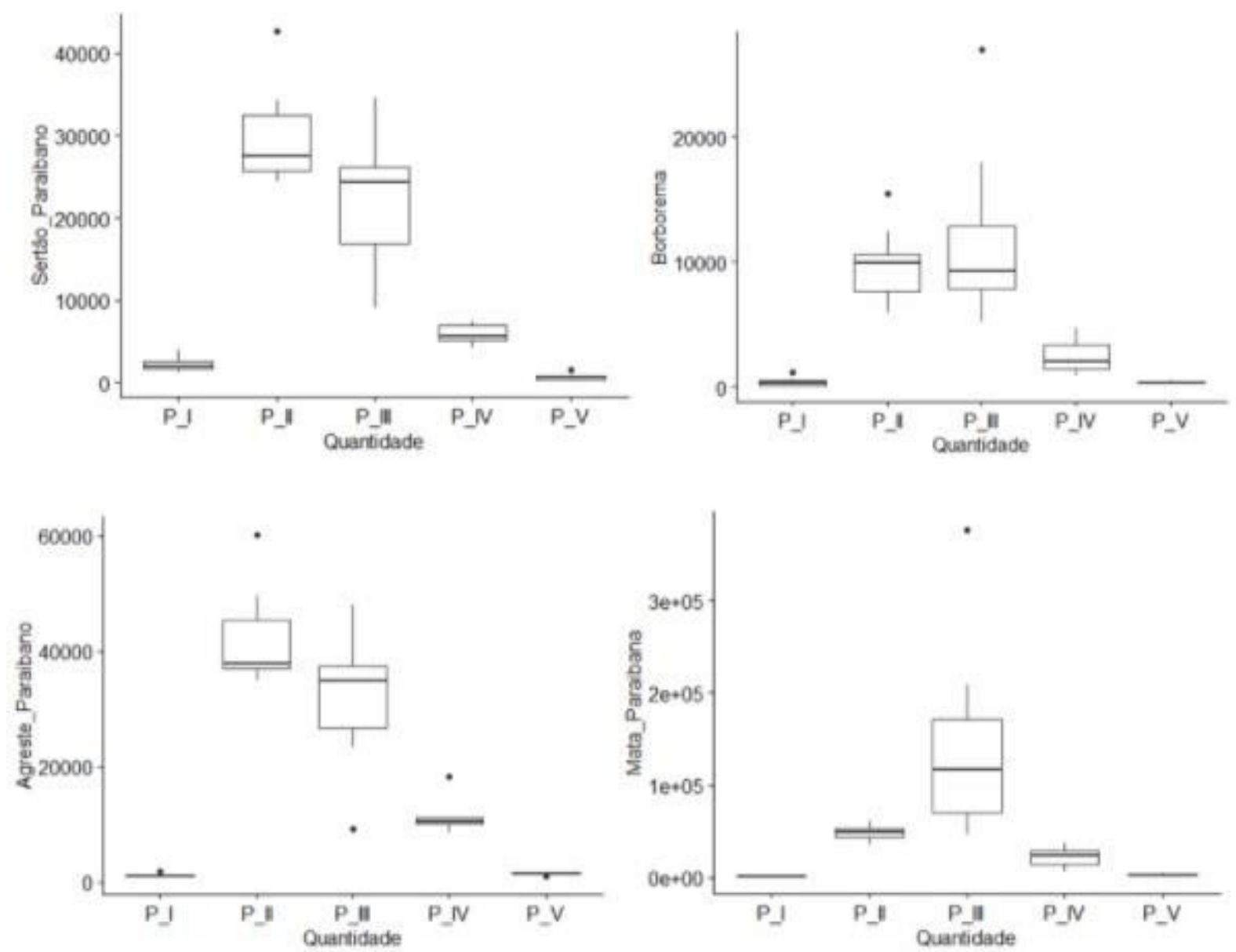

\begin{tabular}{|l|l|}
\hline \multicolumn{2}{|c|}{ Legendas } \\
\hline Procedimento: P-I & Acolhimento inicial em CAPS \\
Procedimento: P-II & Atendimento individual em CAPS \\
Procedimento: P-III & Atendimento em grupo em CAPS \\
Procedimento: P-IV & Atendimento familiar em CAPS \\
Procedimento: P-V & Atendimento domiciliar em CAPS \\
\hline
\end{tabular}

Fonte: Autoria própria.

Para a mesorregião do Sertão, observa-se que a quantidade de atendimentos individuais apresenta apenas um valor acima de 40000, já os atendimentos em grupo tiveram a maior dispersão entre os procedimentos realizados e os atendimentos domiciliares a menor dispersão. Mesmo com a 
presença de um outlier (valor atípico), a quantidade deatendimentos individuais é um dos procedimentos de maior demanda nos CAPS da região. Quanto à Borborema, observa-se um valor elevado na quantidade de atendimentos em grupo, com uma dispersão relativamente maior que os outros procedimentos no decorrer dos anos, seguido pelo número de atendimentos individuais e atendimentos familiares respectivamente.

Nota-se a presença de um ano atípico para os valores de atendimentos individuais eatendimentos em grupo.

Por sua vez, a faixa do Agreste apresenta uma das maiores quantidades absolutas de atendimentos no CAPS, mas esse número parece ser proporcional à população da região que também é uma das mais elevadas. Os atendimentos em grupo e os individuais e em grupo sãoos que apresentam as maiores quantidades e dispersões. Os atendimentos individuais apresentam um valor atípico acima do esperado enquanto os atendimentos em grupo apresentam um valor atípico abaixo do esperado. A zona da Mata possui a maior população dentre todas as mesorregiões, apenas os municípios que compõem a microrregião de João Pessoa já são suficientes para somar 1.159.054 pessoas. Com exceção dos atendimentos em grupo, quantidade de procedimentos realizados nos CAPS da região demonstra pouquíssima dispersão, o que aponta para uma constância no decorrer dos anos. Os atendimentos em grupo da Mata Paraibana também aparecem de forma um pouco simétrica, o valor máximo foi puxado por um ponto extremo, mostrando que a Mata Paraibana tem pontos extremos por causa das grandes cidades. Em todos os procedimentos o PI e PV apresentaram uma baixa variabilidade. O procedimento PIII apresenta observações destoantes das demais e podem ser considerados como outliers ou valores atípicos. Houve uma pequena dispersão na Mata Paraibana em relação ao P II, ou seja, os valores não apresentam uma grande variação em torno da mediana. 


\section{DISCUSSÃO}

Encontram-se evidências na literatura que demonstram que as regiões Sul e Sudeste contam com maior capacidade econômica e melhor acesso aos serviços de saúde, enquanto asregiões Norte e Nordeste apresentam uma baixa significativa nos mesmos indicadores, ainda em fase de implantação das redes assistenciais (ONOCKO-CAMPOS et al., 2018). No Nordeste, estados e municípios apresentam estágios diferentes de consolidação e inovação das suas redes de assistência, fato que pode ser observado nos dados atualizados sobre as atividades dos CAPS na Paraíba. As quantidades registradas de cada procedimento realizado no CAPS são bastante alavancadas pela presença de centros urbanos como as microrregiões de Campina Grande no Agreste e João Pessoa na Zona da Mata. Especialmente no caso de João Pessoa, capital do estado, a atividade dos CAPS no decorrer dos anos mostra-se bastante consistente no decorrer dos anos, o que aponta para presença de uma rede assistencial mais consolidada.

As diferenças aqui observadas entre as respectivas faixas populacionais consideradas no presente estudo evidenciam a existência de propostas de cuidado psicossocial próprias e distintas, respondendo ao quadro de demandas específicas aos contextos comunitários da realidade social em que cada CAPS encontra-se inserido, conforme indicado pela bibliografia de referência (ONOCKO-CAMPOS et al., 2018). As altas dispersões presentes em procedimentos como os atendimentos em grupo em todas as mesorregiões da Paraíba demonstra uma mudança nas estratégias de saúde com o avanço da rede em cada município, sendo a baixa inserção da rede assistencial nas áreas menos populosas do estado passível de ser justificada em função do volume relativamente reduzido de investimentos financeiros direcionados ao cuidado à saúde mental nestas regiões. O Brasil investe apenas cerca de $2,5 \%$ dos seus recursos destinados à saúde em saúde mental, o que é proporcionalmente baixo se considerada a realidade epidemiológica vivida neste âmbito (ONOCKO-CAMPOS et al., 2018), em especial após à crise de saúde mundial proporcionada pela pandemia do vírus Sars-CoV-2, uma vez que a rede de 
atenção à saúde ainda encontra-se em fase de adequação às novas limitações e demandas sociais desta realidade sanitária (NABUCO, OLIVEIRA \& AFONSO, 2020).

Embora tenha sua gestão vinculada à Secretaria de Assistência à Saúde do Ministério da Saúde, o banco de dados do Sistema de Informações Ambulatoriais do Departamento de Informações do Sistema Único de Saúde - SIA/DATASUS é alimentado através do esforço conjunto das diversas Secretarias Estaduais e Municipais de Saúde da Federação. Cabe destacar, portanto, a atual condição de fragilidade e de baixa fidedignidade dos dados qualificados enquanto um verdadeiro desafio logístico e administrativo que dificulta o andamento dos processos de planejamento, desenvolvimento e execução das estratégias de gestão no âmbito da saúde pública (SALES et al., 2019). Apesar dos esforços corretivos empregados, sobretudo, através de arranjos organizativos das ações e serviços de saúde por meio da integração de múltiplos sistemas de suporte tecnológico e logístico, o atual sistema de atenção à saúde ainda é majoritariamente adotado de forma episódica, reativa e fragmentada (OLIVEIRA, 2016).

De fato, o caráter fragmentado da organização infraestrutural do SUS reitera a relevância dos debates e esforços empreendidos no sentido de fomentar o gradativo processo de transição dos sistemas, plataformas e rotinas internas para uma realidade cada vez mais dinâmica e digital, desburocratizando o atual modelo de alimentação dos dados através da implementação de recursos tecnológicos que possibilitem sua atuação como um sistema em rede (MENDES, 2012). A incorporação de atualizações tecnológicas na rotina dos protocolos de trabalho da rede de atenção básica, conquanto vise propiciar o avanço na gestão em termos de agilidade, transparência e seguridade dos dados, tende colidir-se com uma cultura organizacional fortemente pré-estabelecida que, majoritariamente, não se encontra necessariamente afinada com os novos paradigmas da realidade digital (SALES et al., 2019).

Nesse sentido, torna-se fundamental que sejam planejadas e executadas políticas extensivas de qualificação e atualização profissional em todos os níveis de atuação do SUS, com foco na integralidade das práticas de cuidado, do acolhimento aos usuários, na prestação de contas e controle financeiro e, em especial, no 
manejo qualificado das informações aserem repassadas para os bancos de dados governamentais, no sentido de buscar garantir uma melhor transição para o aparelhamento tecnológico priorizando-se a eficiência, a transparência e humanização dos serviços (OLIVEIRA, 2016).

Para além de uma possível solução de viés tecnológico com impactos na cultura organizacional da gestão de trabalho, sublinham-se outros desafios que comprometem de forma significativa a consolidação da plena capacidade de atendimento e de resposta ao volume cada vez maior de demandas sociais que deságuam no SUS, como a insuficiência de recursos humanos e a baixa valorização salarial das equipes multiprofissionais de saúde e demais trabalhadores comprometidos cotidianamente com a rede de atenção básica, sendo este um evidente sinal de uma gestão pública ineficiente e da baixa participação popular nos espaços de discussão, de controle e de fiscalização social (MENDES, CARNUT \& GUERRA, 2018).

Destarte, considerando a importância dos serviços de saúde mental vinculados à inserção social dos CAPS enquanto dispositivos políticos voltados à manutenção da garantia do direito à dignidade humana e qualidade de vida para a população em seus contextos comunitários, sublinha-se a alarmante situação atual de congelamento dos gastos públicos da União para o repasse de verbas federais destinadas ao SUS por um período de 20 anos, posto em prática através da promulgação da Emenda Constitucional 95 (PEC-95/2016) que prevê uma remodelação no modelo de financiamento público dos serviços de saúde que, na prática, representa um grave retrocesso institucional materializado pelo sufocamento das despesas primárias do SUS e seu evidente sucateamento (MENDES, CARNUT \& GUERRA, 2018).

Apesar dos inúmeros desafios e entraves à aplicação eficiente dos recursos e ações oferecidas ao atendimento das demandas de saúde mental, o SUS, através do modelo estruturado pela Rede de Atenção Psicossocial e, em especial, materializado pelas equipes multidisciplinares dos NASF e dos CAPS, permanece ocupando um lugar de referência estratégica e de protagonismo internacional no âmbito da saúde pública, garantindo a promoção de saúde pública de forma universal e integralizada, com foco na orientação, prevenção e manutenção da 
qualidade de vida dos seus usuários (SANTOS, 2018). Nesse sentido, apesar do elevado risco de subnotificação advinda da ausência de leis que regulamentem o processo de adição de informações no sistema, o DATASUS disponibiliza o acesso gratuito, equânime e transparente aos dados quantitativos que podem sustentar e fomentar as tentativas de análise situacional necessárias à boa execução dos processos decisórios através de uma lógica baseada em evidências, sendo, portanto, uma peça-chave na elaboração de quaisquer estratégias de ação em saúde (MACHADO, 2017).

\section{CONSIDERAÇÕES FINAIS}

A proposta de trabalho levantada no presente estudo voltou-se à tentativa de compreensão crítica acerca das prováveis inter-relações estatísticas latentes entre os diversos serviços de saúde mental disponibilizados pelos CAPS das 4 macrorregiões do estado da Paraíba, inseridos na Rede de Atenção Psicossocial que compõe o Sistema Único de Saúde. Têm-se sublinhada, portanto, a elevada pertinência de propostas de pesquisas que não desconsiderem os múltiplos determinantes político-econômicos envolvidos no processo de constituição do entendimento coletivo acerca dos fenômenos de saúde mental e adoecimento psíquico.

Portanto, aqui reitera-se de maneira contundente a defesa do SUS enquanto pauta política fundamental à consolidação de um Estado de Direito que, de fato, se proponha a garantir constitucionalmente o acesso amplo e democrático da população à serviços de saúde humanizados, especializados e de alta qualidade, sobretudo com foco nas estratégias de promoção e prevenção. Para tanto, destacase a necessidade de que sejam incentivadas novas pesquisas científicas de caráter exploratório acerca das reais condições de trabalho e de produção de serviços junto aos profissionais inseridos nos espaços de atuação da atenção primária, em especial os CAPS, de forma que as carências e demandas estruturais possam ser 
devidamente evidenciadas e sanadas no plano da gestão pública de saúde em todas as esferas governamentais.

\section{REFERÊNCIAS BIBLIOGRÁFICAS}

AMARANTE, P. Uma aventura no manicômio: a trajetória de Franco Basaglia. História, Ciências, Saúde - Manguinhos. v. 1, n.1, p. 61-77. 1994.

CAMPOS, F. C. B.; \& GUARIDO, E. L. O Psicólogo no SUS: suas práticas e as necessidades de quem o procura. In: Spink, M. J. P. (Org.) A psicologia em diálogo com o SUS: prática profissional e produção acadêmica. São Paulo: Casa do Psicólogo. p. 81-103. 2010.

GODOI, L. P. S. Apoio Matricial como ferramenta de articulação entre Atenção Básica e CAPS. Tese de mestrado em saúde pública. Faculdade de Saúde Pública da USP. p. 01-79. São Paulo: 2020.

GOMES, L. M. D. O Núcleo de Apoio à Saúde da Família (NASF) e a Integralidade na Atenção Básica em Saúde Mental em um Município da Região Serrana do Rio de Janeiro. Tese de mestrado profissional em vigilância em saúde. FIOCRUZ. p. 01-81. Rio de Janeiro: 2013.

INSTITUTO BRASILEIRO DE GEOGRAFIA E ESTATÍSTICA - IBGE. Diretoria de Pesquisas, Coordenação de População e Indicadores Sociais. Estimativas da população residente com data de referência $1^{\circ}$ de julho de 2020. Rio de Janeiro: 2020. Acesso em: 20 Fev. 2020.

INSTITUTO BRASILEIRO DE GEOGRAFIA E ESTATÍSTICA - IBGE. Malhas digitais. Rio de Janeiro: 2017. Acesso em: 20 Fev. 2020.

MENDES, E. V. 25 anos do Sistema Único de Saúde: resultados e desafios. Estud. av., São Paulo. v. 27, n. 78, p. 27-34. 2013.

MACHADO, G. C. Avaliação quantitativa da produção ambulatorial em saúde auditiva no município de Salvador-Bahia no período de 2004 a 2016. Rev. Ciênc. Méd. Biol. v. 16, n. 3, p. 323-328. Salvador: 2017.

MENDES, Á.; CARNUT, L.; \& GUERRA, L. D. S. Reflexões acerca do financiamento federal da Atenção Básica no Sistema Único de Saúde. Saúde debate. v. 42, n. spe1, p. 224243. Rio de Janeiro: 2018.

NABUCO, G.; OLIVEIRA, M. H. P. P.; \& AFONSO, M. P. D. O impacto da pandemia pela COVID-19 na saúde mental: qual é o papel da Atenção Primária à Saúde? Rev Bras Med Fam Comunidade. v. 15, n. 42, p. 2532. 2020.

OLIVEIRA, N. R. C. Redes de atenção à saúde: a atenção à saúde organizada em redes. Universidade Federal do Maranhão. UNA-SUS/UFMA. São Luís/MA: 2016.

ONOCKO-CAMPOS, R. T. et al. Atuação dos Centros de Atenção Psicossocial em quatro centros urbanos no Brasil. Rev Panam Salud Publica. v. 42. e113. 2018.

QUEIROZ, T. H. S. Análise histórica dos diferenciais de mortalidade por sexo e mesorregiões da Paraíba, 1980-2010. Trabalho de Conclusão de Curso em Ciências Atuariais. Centro de 
Ciências Sociais Aplicadas da UFPB. João Pessoa/PB: 2017.

R CORE TEAM. R: A language and environment for statistical computing. R Foundation for Statistical Computing. Vienna, Austria: 2019. Disponível em: https://www.R- project.org/.

RIBEIRO, J. L. P. A Psicologia da Saúde. In: Alves, R. F. (Org.) Psicologia da saúde: teoria, intervenção e pesquisa [online]. Campina Grande: EDUEPB. p. 23-59. 2011.

SALES, O. P., et al. O Sistema Único de Saúde: desafios, avanços e debates em 30 anos de história. Revista Humanidades e Inovação. v. 6, n. 17, p. 54-65. 2019.

SANTOS, L. SUS 30 anos: um balanço incômodo? Ciência \& Saúde Coletiva. v. 23, p. $2043-$ 2050. 2018.

BOUGEARD, S., \& Dray, S (2018). "Supervised Multiblock Analysis in R with the ade4 Pac kage." Journal of Statistical Software_. URL: https://doi.org/10.18637/jss.v086.i01.

TAIYUN, W. \& VILIAM, S. R package "corrplot": Visualization of a Correlation Matrix (Version 0.84). 2017. Available from https://github.com/taiyun/corrplot. 